\title{
Clinical applicability of biologically effective dose calculation for spinal cord in fractionated spine stereotactic body radiation therapy
}

\author{
Seung Heon Lee ${ }^{1}$, Kyu Chan Lee ${ }^{1}$, Jinho Choi ${ }^{1}$, So Hyun Ahn¹, Seok Ho Lee ${ }^{1}$, Ki Hoon Sung ${ }^{1}$, \\ Se Hee Kil2 \\ ${ }^{1}$ Department of Radiation Oncology; ${ }^{2}$ Gachon Medical Research Institute, Gachon University Gil Medical Center, Republic of
Korea
}

Radiol Oncol 2015; 49(2): 185-191.

Received 7 November 2014

Accepted 5 January 2015

Correspondence to: Kyu Chan Lee, M.D., Ph.D., Department of Radiation Oncology, Gachon University Gil Medical Center 21, 774 beon-gil, Namdong-daero, Namdong-gu, Incheon, 405-460, Republic of Korea. Phone: +82 32460 8050; Fax: +82 324603009 ; E-mail: kyu22@gilhospital.com

Disclosure: No potential conflicts of interest were disclosed.

Background. The aim of the study was to investigate whether biologically effective dose (BED) based on linearquadratic model can be used to estimate spinal cord tolerance dose in spine stereotactic body radiation therapy (SBRT) delivered in 4 or more fractions.

Patients and methods. Sixty-three metastatic spinal lesions in 47 patients were retrospectively evaluated. The most frequently prescribed dose was $36 \mathrm{~Gy}$ in 4 fractions. In planning, we tried to limit the maximum dose to the spinal cord or cauda equina less than $50 \%$ of prescription or $45 \mathrm{~Gy}_{2 / 2}$. BED was calculated using maximum point dose of spinal cord.

Results. Maximum spinal cord dose per fraction ranged from 2.6 to $6.0 \mathrm{~Gy}$ (median $4.3 \mathrm{~Gy}$ ). Except 4 patients with $52.7,56.4,62.4$, and $67.9 \mathrm{~Gy}_{2 / 2}$, equivalent total dose in 2-Gy fraction of the patients was not more than $50 \mathrm{~Gy}_{2 / 2}(12.1-$ 67.9, median 32.0). The ratio of maximum spinal cord dose to prescription dose increased up to $82.2 \%$ of prescription dose as epidural spinal cord compression grade increased. No patient developed grade 2 or higher radiationinduced spinal cord toxicity during follow-up period of 0.5 to 53.9 months.

Conclusions. In fractionated spine SBRT, BED can be used to estimate spinal cord tolerance dose, provided that the dose per fraction to the spinal cord is moderate, e.g. < $6.0 \mathrm{~Gy}$. It appears that a maximum dose of up to $45-50 \mathrm{~Gy} / 2$ to the spinal cord is tolerable in 4 or more fractionation regimen.

Key words: biologically effective dose; spine stereotactic body radiation therapy; spinal cord; tolerance dose; linear quadratic model

\section{Introduction}

Stereotactic body radiation therapy (SBRT) has been increasingly applied to the management of spinal metastases with encouraging clinical results of rapid and durable pain relief., ${ }^{1,2}$ SBRT is also effective for treating radio-resistant metastatic tumours, such as, renal cell carcinoma or malignant melanoma. ${ }^{3,4}$

The spinal cord is the major dose-limiting tissue in spine SBRT. In conventionally fractionated radiation therapy, the tolerance dose for the spinal cord has been reported to be 50 Gy for cord lengths of 5 and $10 \mathrm{~cm}$, and $47 \mathrm{~Gy}$ for $20 \mathrm{~cm}$, given a probability of myelopathy of less than $5 \%$ within 5 years. ${ }^{5}$ Schultheiss reported that the probabilities of myelopathy were $0.03 \%$ and $0.2 \%$ at $45 \mathrm{~Gy}$ and $50 \mathrm{~Gy}$, respectively. ${ }^{6}$ For single fraction SBRT, Ryu et al. reported a partial volume tolerance of the human spinal cord of at least $10 \mathrm{~Gy}$ to $10 \%$ of the spinal cord volume when spinal cord volume was defined from $6 \mathrm{~mm}$ above and to $6 \mathrm{~mm}$ below the 
TABLE 1. Patient and tumour characteristics

\begin{tabular}{|c|c|}
\hline Age (year, median) & $33-86(56)$ \\
\hline \multicolumn{2}{|l|}{ Histology (person) } \\
\hline Lung & 13 \\
\hline Colorectal & 11 \\
\hline Breast & 6 \\
\hline Pancreas & 3 \\
\hline Hepatocellular & 3 \\
\hline Stomach & 2 \\
\hline Cholangiocarcinoma & 2 \\
\hline Prostate & 2 \\
\hline Renal cell & 1 \\
\hline Other & 4 \\
\hline \multicolumn{2}{|l|}{ Spine level (lesion) } \\
\hline Cervical & 3 \\
\hline Cervicothoracic & 4 \\
\hline Thoracic & 37 \\
\hline Thoracolumbar & 5 \\
\hline Lumbar & 14 \\
\hline \multicolumn{2}{|c|}{ Number of involved spine segments per PTV (lesion) } \\
\hline 1 & 24 \\
\hline 2 & 17 \\
\hline 3 & 16 \\
\hline 4 & 3 \\
\hline 5 & 3 \\
\hline Tumour volume (cc, median) & $\begin{array}{c}1.0-176.7 \\
(21.0)\end{array}$ \\
\hline PTV volume (cc, median) & $\begin{array}{c}17.9-340.8 \\
(59.1)\end{array}$ \\
\hline \multicolumn{2}{|c|}{ Number of treated sites per patient (person) } \\
\hline 1 & 35 \\
\hline 2 & 8 \\
\hline 3 & 4 \\
\hline \multicolumn{2}{|l|}{ ESCC grade $^{20}$ (lesion) } \\
\hline 0 & 14 \\
\hline la & 15 \\
\hline $1 \mathrm{~b}$ & 9 \\
\hline lc & 5 \\
\hline 2 & 18 \\
\hline 3 & 2 \\
\hline
\end{tabular}

ESCC = epidural spinal cord compression; PTV = planning target volume treatment target. ${ }^{7}$ Sahgal et al. suggested 10 Gy as a maximum safe threshold for single fraction SBRT to the thecal sac. ${ }^{8}$

In fractionated spine SBRT, however, variable dose schedules are applied and no reliable dose comparison method has been established for the target or the spinal cord. Since Fowler first proposed the term 'biologically effective dose' based on linear-quadratic (LQ) cell survival model in 1989, BED has been used to compare the biologic effects of various radiotherapy schedules. ${ }^{9}$ However, because prescription doses in fractionated spine SBRT are usually between 6 and $10 \mathrm{~Gy}$ per fraction, several authors have argued that the simple application of BED based on LQ model is not appropriate in SBRT. ${ }^{10-18}$ The extrapolations using the LQ model beyond 5-6 Gy per fraction are likely to lack clinically useful precision. ${ }^{19}$

Modern linear accelerator based stereotactic radiotherapy technology using a fine multileaf collimator of $2.5 \mathrm{~mm}$ thickness could deliver highly conformal radiation to the target while sparing the spinal cord with the merit of a steep dose gradient just outside the target. The irradiated dose to the spinal cord can be more strictly limited and is usually much lower than the prescription dose in fractionated spine SBRT. We hypothesized that if maximum doses per fraction to the spinal cord are less than 6 Gy, BED based on LQ model could be used to estimate spinal cord tolerance dose in fractionated spine SBRT. We usually implemented fractionated spine SBRT in 4 or 5 fractions to avoid complications such as radiation-induced myelopathy and vertebral compression fracture.

To determine if BED based on LQ model can be used to estimate spinal cord tolerance dose in fractionated spine SBRT of 4 or more fractionation regimen, the plans used for actual fractionated SBRT at our institution were analysed retrospectively and clinical outcomes, including complications, were investigated.

\section{Patients and methods}

Sixty-three metastatic spinal lesions in 47 patients were treated by spine SBRT between January 2010 and March 2014. Median patient age was 56 (range $33-86)$ and 27 patients $(57.4 \%)$ were male. The thoracic spine was the most frequent site for treatment. Of the 63 lesions, 41 lesions involved single or two contiguous spine segments. When categorized with the epidural spinal cord compression (ESCC) grading system, $43.5 \%$ of the lesions belonged to 
grade 1c (deformation of the thecal sac with spinal cord abutment), 2 (spinal cord compression, but with cerebrospinal fluid [CSF] visible around the cord) or 3 (spinal cord compression, no CSF visible around the cord) (Table 1). ${ }^{20}$ No patients received surgical intervention, vertebroplasty or kyphoplasty, prior to spine SBRT.

All patients underwent $\mathrm{CT}$ simulation with an appropriate immobilization technique to obtain SBRT planning images. To delineate targets and spinal cords, T2-weighted and gadolinium contrast T1-weighted MRI sequences with a $3 \mathrm{~mm}$ slice thickness including at least one vertebral body above and below the target were obtained and fused to the planning CT image using iPlan software (version 4.1, BrainLAB, Germany). Gross tumour volume (GTV) included gross visible tumour in spine, paraspinal, or epidural area on MR or enhanced planning CT images. Planning target volumes (PTV) were derived from GTVs by encompassing involved vertebral body and including anterior and/or posterior elements of the spine depending on the location of metastatic lesions as described in RTOG 0631. ${ }^{21}$ Median PTV volume was $65.6 \mathrm{cc}$ (range, 17.9-340.8 cc). The spinal cord was contoured starting from $6 \mathrm{~mm}$ above the superior of the PTV to $6 \mathrm{~mm}$ below the inferior of the PTV. However, the spinal cord was always excluded from the PTV, with a 1-2 $\mathrm{mm}$ free margin if the GTV did not abut onto the cord. Other organs, such as heart, lungs, oesophagus, large vessels, trachea, liver, and kidneys were delineated depending on tumour vertebral level.

The most frequently prescribed dose was $36 \mathrm{~Gy}$ in 4 fractions, followed by 40 Gy in 5 fractions (Table 2). The requirement for clinical implementation was $>80 \%$ of the prescription dose to $>90 \%$ of the PTV, or a mean PTV dose $>95 \%$ of prescription. The spinal cord dose was converted to equivalent total dose in 2-Gy fraction $\left(\mathrm{EQD}_{2}\right)$ using the following formula provided below. This model was derived from the LQ model assuming an $\alpha / \beta$ ratio of 2 for the late effect of spinal cord.

$\mathrm{EQD}_{2}\left(\mathrm{~Gy}_{2 / 2}\right)=$ Total dose $\mathrm{x}\left(\frac{\text { Dose per fraction }+\alpha / \beta}{2+\alpha / \beta}\right)$

We tried to limit the maximum dose to the spinal cord or cauda equina less than $50 \%$ of prescription or $45 \mathrm{~Gy}_{2 / 2}$. Maximum dose per fraction to the spinal cord of each plan was investigated.

All patients were treated using the Novalis $\mathrm{Tx}^{\mathrm{TM}}$ (Varian, USA) equipped with a $2.5 \mathrm{~mm}$ multileaf collimator. Thirty-five patients received SBRT to
TABLE 2. Prescription dose to planning target volume and maximum dose to spinal cord

\begin{tabular}{lcc}
\hline Total dose / fractions & Number & $\begin{array}{c}\text { Maximum dose } \\
\text { to spinal cord } \\
\left(\mathrm{EQD}_{2}, \mathrm{~Gy}_{2 / 2}\right)\end{array}$ \\
\hline 26.0 Gy / 4 fractions & 1 & 24.8 \\
28.0 Gy / 4 fractions & 1 & 25.4 \\
30.0 Gy / 4 fractions & 1 & 52.7 \\
32.0 Gy / 4 fractions & 2 & $25.5-56.4$ \\
36.0 Gy / 4 fractions & 23 & $12.1-67.9$ \\
40.0 Gy / 4 fractions & 1 & 24.3 \\
44.0 Gy / 4 fractions & 2 & $38.9-44.4$ \\
32.5 Gy / 5 fractions & 6 & $16.6-43.0$ \\
35.0 Gy / 5 fractions & 4 & $25.7-49.0$ \\
40.0 Gy / 5 fractions & 19 & $24.2-41.1$ \\
42.5 Gy / 5 fractions & 1 & 38.7 \\
42.0 Gy / 6 fractions & 2 & $39.8-62.4$ \\
\hline
\end{tabular}

$\mathrm{BED}=$ biologically effective dose; $\mathrm{EQD}_{2}=$ equivalent dose in 2-Gy fractions with an $\alpha / \beta$ ratio of 2

a single treatment site spanning one to five vertebral segments. Eight patients received SBRT to 2 separate sites and 4 patients to 3 sites. Re-SBRT was performed in one patient with hepatocellular carcinoma at 5 months after initial SBRT because of the recurrence of severe pain due to tumour progression.

Patients were followed up clinically and radiographically at 1- to 3-month intervals. A visual analogue scale (VAS) was used to measure pain before and after treatment. Symptomatic responses were scored as defined by RTOG $0631 .{ }^{21}$ All available follow up MRIs were reviewed to assess radiographic responses. Radiologic local failure was defined as local tumour growth by MRI. Late complications were scored as described by Common Toxicity Criteria for Adverse Events, version 4.0. Overall survival was estimated by the Kaplan-Meier method. The relationship between radiologic local failure and variable candidate risk factors such as spinal cord to tumour distance, spinal cord to PTV distance, minimum dose administered to tumour, and tumour volume was analyzed by the MannWhitney U-test. The retrospective study was approved by the institutional review board committee and was according to the Helsinki Declaration.

\section{Results}

Inhomogeneous dose distributions inside the spinal cord and very steep dose gradients around it were observed (Figure 1A). Approximately 10\% decrease of dose per millimetre was observed from 
(A)

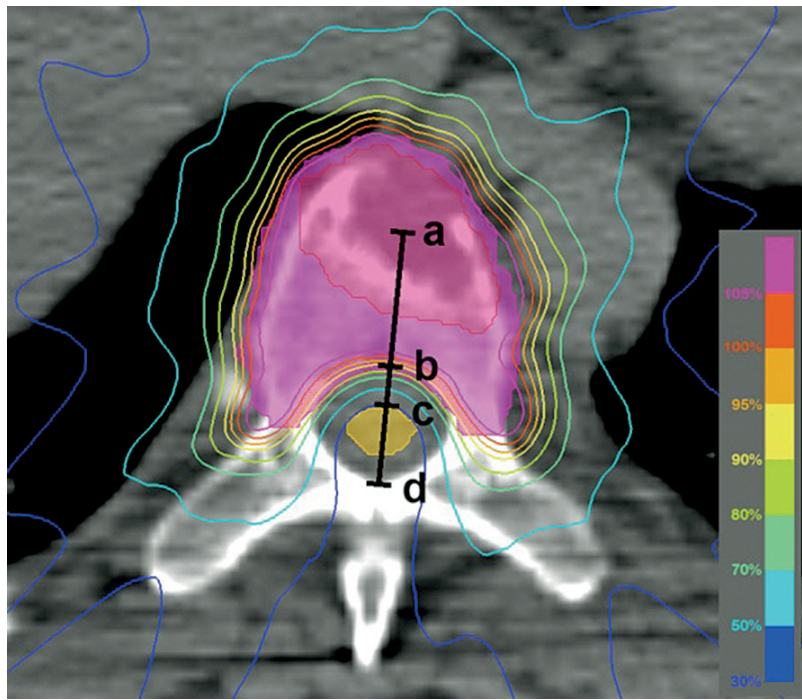

(B)
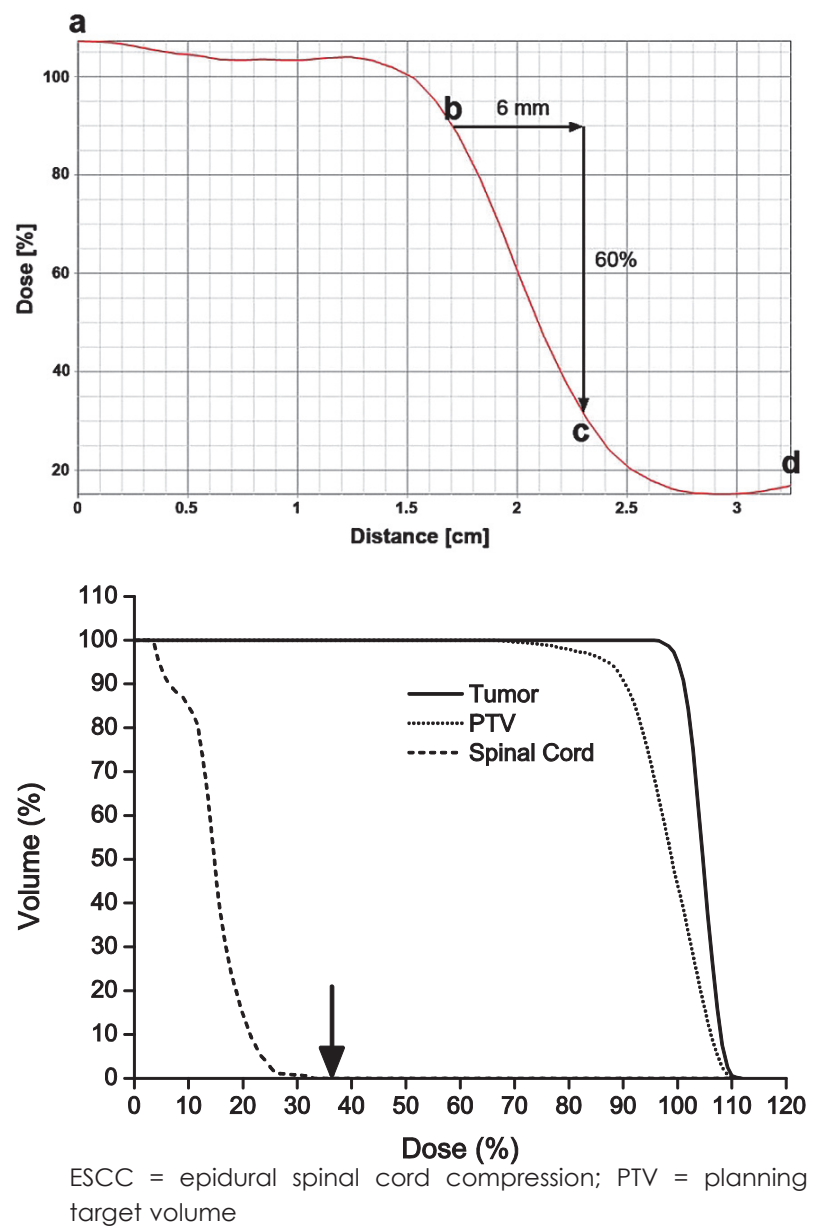

FIGURE 1. An example of dose distribution in a patient with disease of the T7 vertebral body only (ESCC grade ${ }^{20}$ 0). (A, B) Dose profile from the center of the tumour (a) to the posterior edge of the thecal sac (d). Note that the dose gradient around the spinal cord in this case is steepest between (b) and (c), where $90 \%$ and $30 \%$ isodose lines, and is approximately $10 \%$ per millimeter. (C) Dose volume histogram of the patient shows much difference of the doses to target and spinal cord. The maximum dose per fraction to the spinal cord (arrow) was 37\% (3.0 Gy) of the prescription dose (9.0 Gy).
PTV margin to the surface of spinal cord (Figure 1B). Maximum dose to spinal cord was much lower than prescription dose (Figure 1C). According to PTV shape and spinal cord proximity, maximum spinal cord doses varied from 10.5 Gy to $33.2 \mathrm{~Gy}$ (median 20.2 Gy). When they were divided by corresponding fraction numbers, the maximum spinal cord dose per fraction ranged from 2.6 to $6.0 \mathrm{~Gy}$ (median $4.3 \mathrm{~Gy}$ ). Maximum $\mathrm{EQD}_{2}$ to spinal cords ranged from 12.1 to $67.9 \mathrm{~Gy}_{2 / 2}$ (median $32.0 \mathrm{~Gy}_{2 / 2}$ ). Six cords were administered more than $45 \mathrm{~Gy}_{2 / 2}$ and doses of them were 48.0, 49.0, 52.7, 56.4, 62.4, and $67.9 \mathrm{~Gy}_{2 / 2}$, respectively. The ratio of maximum spinal cord dose to prescription dose increased up to $82.2 \%$ of prescription dose as the ESCC grade increased (Table 3).

Median follow-up period was 7.1 months (0.5-53.9 months) and median overall survival was 10.2 months. During follow-up, 26 patients succumbed to systemic disease progression. VAS results were available for 46 of the 63 lesions. Mean VAS declined from 7.8 before to 2.7 after SBRT. Complete response was achieved for 10 lesions and partial response for 28 lesions.

Follow-up MRIs were available for 27 lesions. Radiologic local failure occurred in 4 lesions $(14.8 \%$, Table 4$)$. All patients in the radiologic local failure group (4 patients) had spinal cord compression (ESCC grade 2) before SBRT. In the non-failure group (23 patients), distances between spinal cords and tumours or PTVs ranged from 0 to $14 \mathrm{~mm}$ (median 2.3) or from 0 to $3.5 \mathrm{~mm}$ (median 1.0), respectively. Spinal cord to tumour distance $(\mathrm{p}<0.001)$, spinal cord to PTV distance $(p=0.003)$, and minimum dose administered to tumours $(p=0.040)$ in the local failure group were significantly smaller than those in the non-failure group. No intergroup difference of tumour volumes was observed. There has been no grade 2 or more radiation-induced spinal cord toxicity during follow up period up to 53.9 months. There were three compression fractures (3 of $27,11.1 \%$ ); two resulted from progressions of existing fractures and the other was a new fracture. Fractures occurred at 2, 3, and 4 months after treatment, respectively.

\section{Discussion}

BED based on the LQ model has been widely accepted for the comparisons of doses administered in different treatment schedules in treating with conventional multifractionated irradiation. However, dose comparisons for fractionated spine 
SBRT based on simple BED calculations should be approached with caution. First, BED was developed and validated based on homogenous radiation dose distributions in irradiated areas. ${ }^{22,23}$ The radiation dose administered to the spinal cord in SBRT is intrinsically inhomogeneous because it is performed with an intensity modulated radiation beam. The data regarding the tolerance of the rat cervical spinal cord suggested that small volume of rat spinal cord tolerates a greater dose compared to homogeneous radiation. ${ }^{24,25}$ Although those observations were not found in swine model, the tolerance dose of spinal cord for partial-volume irradiation closely resembled that for rats, mice and guinea pigs receiving uniform spinal cord irradiation. ${ }^{26}$ Therefore, estimated spinal cord tolerance using BED calculation for partial-volume irradiation seems to be more conservative than, or at least comparable to that for uniform irradiation.

Second, BED has been based on the data of conventional fraction size. However, in fractionated SBRT, prescription dose per fraction is relatively high, usually 6-20 Gy. Fundamental arguments have arisen as to whether the LQ model is a valid method for assessing BED when doses per fraction are high. Brenner et al. reported that the LQ model is reasonably well validated experimentally and theoretically up to about 10 Gy per fraction, and suggested that its use is reasonable up to about 18 Gy per fraction. ${ }^{27}$ However, several authors have argued to the contrary. Iwata et al. studied the applicability of the LQ model for dose conversion in high dose per fraction radiotherapy using cell survival data for V79 Chinese hamster lung fibroblasts and EMT6 mouse mammary sarcoma cells. ${ }^{14}$ It was found that the LQ model fitted relatively well at doses of 5 Gy or less as compared with the repairable-conditionally repairable model and the multi-target model. Timmerman et al. proposed a universal survival curve that hybridizes the LQ model survival curve for the low-dose range and the multi-target model asymptote for the high-dose range. ${ }^{15}$ They reported a transition dose at which the LQ model smoothly transits to the terminal asymptote of the multi-target model. The transition dose calculated using 12 non-small-cell lung cancer (NSCLC) cell lines was $6.2 \mathrm{~Gy}$, which means that LQ model may not be applicable for dose ranges of more than 6.2 Gy. Recently, Song et al. argued that the usefulness of the LQ model is likely to be limited when tumours are treated with high dose per fraction, usually more than $10 \mathrm{~Gy}$, because LQ model and other modified-LQ models are based on the assumption that radiation-induced cell death in
TABLE 3. Spinal cord dose classified using the epidural spinal cord compression (ESCC) grading system ${ }^{20}$

\begin{tabular}{cclll}
\hline Grade & lesions & $\begin{array}{l}\mathrm{D}_{\max } \\
(\mathrm{Gy}, \text { median) }\end{array}$ & $\begin{array}{l}\mathrm{EQD}_{2 \text { max }} \\
\left(\mathrm{Gy}_{2 / 2}, \text { median }\right)\end{array}$ & $\begin{array}{l}\mathrm{D}_{\max } / \text { prescription } \\
\text { dose }(\%, \text { median) }\end{array}$ \\
\hline 0 & 14 & $12.0-25.5(18.3)$ & $15.0-38.9(29.6)$ & $33.4-60.7(50.5)$ \\
1a & 15 & $10.5-25.3(20.8)$ & $12.1-52.7(32.0)$ & $29.1-84.3(52.0)$ \\
1b & 9 & $13.9-23.4(19.3)$ & $16.6-44.4(33.4)$ & $40.3-56.2(53.4)$ \\
1c & 5 & $16.3-22.3(17.5)$ & $24.8-36.1(28.0)$ & $45.9-63.8(57.1)$ \\
2 & 18 & $16.5-33.2(21.8)$ & $24.2-67.9(36.1)$ & $43.9-81.1(58.4)$ \\
3 & 2 & $21.4-26.3(23.9)$ & $39.3-56.4(47.9)$ & $59.4-82.2(70.8)$ \\
\hline
\end{tabular}

$D_{\max }=$ maximum dose to spinal cord; $E E_{2}$ max $=$ maximum equivalent dose in 2-Gy fractions with an $\mathrm{a} / \beta$ ratio of $2 ; \mathrm{ESCC}=$ epidural spinal cord compression

TABLE 4. Distances from spinal cord to tumour or planning target volume (PTV), minimum tumour doses, and tumour volumes according to radiologic local failure status

\begin{tabular}{lccc}
\hline & $\begin{array}{c}\text { Failure group } \\
\text { (4 lesions) }\end{array}$ & $\begin{array}{c}\text { Non-failure group } \\
\text { (23 lesions) }\end{array}$ & p-value** \\
\hline $\begin{array}{l}\text { Distance between } \\
\text { SC and tumour } \\
\text { (mm, median) }\end{array}$ & $0 *$ & $0-14.0(2.3)$ & $<0.001$ \\
$\begin{array}{l}\text { Distance between } \\
\text { SC and PTV } \\
\text { (mm, median) }\end{array}$ & $0 *$ & $0-3.5(1.0)$ & 0.003 \\
$\begin{array}{l}\text { Minimum tumour dose } \\
\text { (Gy, median) } \\
\text { Tumour volume } \\
\text { (cc, median) }\end{array}$ & $15.4-23.8(20.0)$ & $15.3-44.7(25.2)$ & 0.040 \\
\hline
\end{tabular}

SC $=$ spinal cord; $*$ All tumours compressed the spinal cord; ** Statistical significance was determined using the Mann-Whitney U-test

tumours is due solely to DNA strand breaks. They suggested indirect/necrotic cell death as a consequence of vascular damage plays an important role in SBRT. ${ }^{17,18}$ As of now, the applicability of BED based on the LQ model to the high dose per fraction radiation remains a controversial issue.

According to the current radiobiological knowledge as mentioned above, BED based on the LQ model seems to be clinically applicable if the dose is limited to 6 Gy or less, especially for normal tissue, not tumour. With current technical developments, dose to the spinal cord can be maintained at much lower levels than the prescription dose due to the steep dose gradient just outside the target. In the present study, the maximum irradiation dose per fraction to the spinal cord varied from 2.6 to 6.0 Gy (median 4.3 Gy) depending on the PTV shape and its proximity to the spinal cord. Because the dose per fraction to the spinal cord was less than $6.0 \mathrm{~Gy}$, it would be reasonable to estimate spinal cord tolerance dose in fractionated SBRT using BED based on LQ model.

Recently, Sahgal et al. recommended limiting maximum point dose to 23.0 Gy in 4 fractions 
(A)
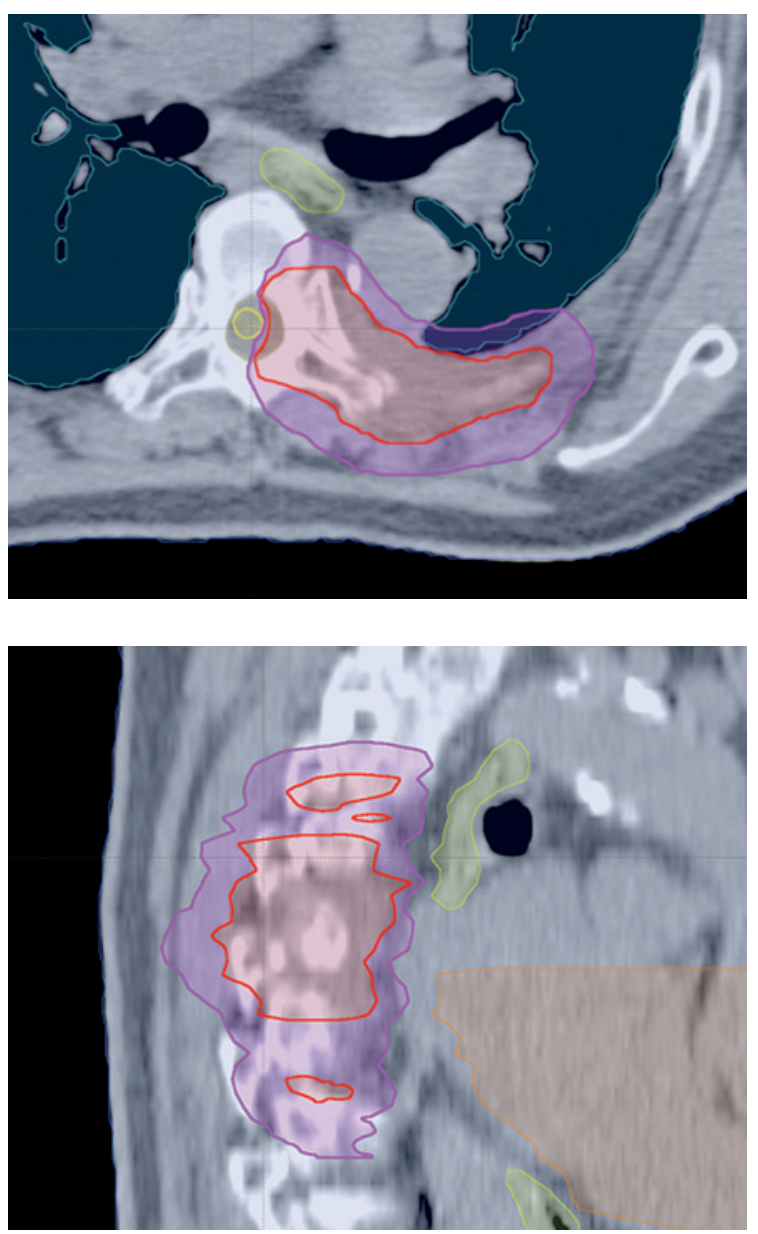

(B)

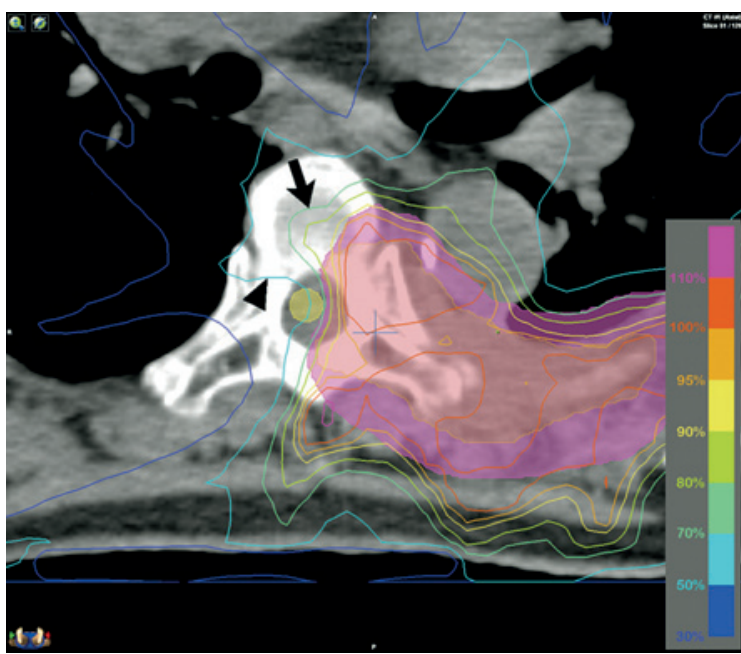

ESCC = epidural spinal cord compression;GTV = gross tumour volume; PTV = planning target volume

FIGURE 2. (A, B) Target volume (GTV: red, PTV: magenta) delineation in a patient with cord compression (ESCC grade 20 2) and paraspinal mass. Five spine segments were involved in the PTV. (C) Dose distribution around the spinal cord. The $70 \%$ (arrow) and $50 \%$ (arrowhead) isodose lines are shown. The maximum spinal cord dose was $33.2 \mathrm{~Gy}$, which was equivalent to $78.9 \%$ of the prescription dose of $42 \mathrm{~Gy}$ in 6 fractions. and $25.3 \mathrm{~Gy}$ in 5 fractions for a risk of radiation myelopathy of less than $5 \% .{ }^{28}$ Dividing the constraint dose by fraction number, maximum point doses per fraction to the thecal sac are $5.75 \mathrm{~Gy}$ and $5.06 \mathrm{~Gy}$, respectively. The calculated $\mathrm{EQD}_{2}$ of thecal sac based on the LQ model for these schedules were $44.6 \mathrm{~Gy}_{2 / 2}$ and $44.7 \mathrm{~Gy}_{2 / 2}$ respectively. These seem to be reasonable because the tolerance dose of spinal cord in conventionally fractionated radiation therapy is $45-50$ Gy with fraction size of 1.8 or 2.0 Gy when full thickness of the cord is irradiated. In Sahgal's data, there was no radiation myelitis after irradiation in 4 or 5 fractions, though the patient number is relatively small, 9 cases. In the present study, except 4 patients, all patients were administered a maximum spinal cord dose less than $50 \mathrm{~Gy}_{2 / 2}$ and no radiation myelopathy was observed among 63 cases.

Gerszten et al. reported that post-SBRT tumour progressions often occurred at the edge of contoured treatment volumes and the overall mean tumour volume of local failure cases was $40 \%$ greater than the average for their series. ${ }^{29}$ Chang et al. also reported that failure at the epidural space adjacent to the spinal cord is a major reason for tumour progression after spine SBRT. ${ }^{10}$ In the present study, minimum tumour dose $(p=0.040)$, which is mainly affected by distance between spinal cord and tumour ( $p<0.001)$ or PTV $(p=0.003)$ appeared to have more influence on local failure. Furthermore, when the tumour did not abut the spinal cord, local failure was not observed even in tumours larger than those in local failure group. These results mean that tumoricidal dose was not delivered to tumour because of the proximity of spinal cord in local failure group. BED calculation has clinical impact on choosing appropriate fraction size and number to deliver optimal tumour dose, especially for the lesions close to spinal cord, while limiting spinal cord dose of less than $45-50 \mathrm{~Gy}_{2 / 2}$.

When a tumour abuts the spinal cord, increasing the number of fractions to deliver potentially tumoricidal dose, with lowering spinal cord BED, might be considered. In the patient shown in Figure 2, there was a large paraspinal tumour mass compressing the spinal cord. By increasing the number of fractions to 6 and decreasing the prescription dose per fraction to the PTV to 7 Gy, we tried to spare the spinal cord delivering 42 Gy to the GTV to improve local control. Although maximum $\mathrm{EQD}_{2}$ of the spinal cord was $62.4 \mathrm{~Gy}_{2 / 2}$, we treated this patient in the palliative setting because local tumour control was important for the quality of life of the patient. 
This study has several limitations; it is a retrospective and single center study, cohort was small, and there was no myelopathy case. Rare but severe events like myelopathy require high patient numbers to evaluate safe tolerance doses and it should not give a false sense of security. Although no myelopathy was observed in 6 patients who were administered a maximum spinal cord dose greater than $45 \mathrm{~Gy}_{2 / 2}$, their survival period was only 1.5-6.4 months. They had multiple metastases in liver or lung and had short life expectancy. Therefore, it is not possible to suggest more doses for spinal cord tolerance above this dose level. However, we believe that the data of our study is important for applying BED calculation for spinal cord tolerance dose in various clinical situations. We plan to conduct a multi-center prospective study with more patients.

In conclusion, BED can be used to estimate spinal cord tolerance dose, provided that the dose per fraction to the spinal cord is moderate, e.g. $<6.0$ Gy in fractionated spine SBRT. Within this dose range it appears that a maximum dose of up to $45-50 \mathrm{~Gy}_{2 / 2}$ to the spinal cord is tolerable. The minimum tumour dose, which is mainly affected by tumour to spinal cord distance, seems to significantly affect local failure. When a tumour abuts or is closely located to the spinal cord, we suggest adjustment of the fractionation schedule based on BED calculations, while maintaining the desirable dose to the target. Randomized controlled dose escalation study is reserved to verify this suggestion.

\section{Acknowledgments}

This work was supported by Gachon University Gil Medical Center (Grant number : 2013-42).

\section{References}

1. Ryu S, Jin R, Jin JY, Chen Q, Rock J, Anderson J, et al. Pain control by imageguided radiosurgery for solitary spinal metastasis. J Pain Symptom Manage 2008; 35: 292-8.

2. Wang XS, Rhines LD, Shiu AS, Yang JN, Selek U, Gning I, et al. Stereotactic body radiation therapy for management of spinal metastases in patients without spinal cord compression: a phase 1-2 trial. Lancet Oncol 2012; 13: 395-402.

3. Balagamwala EH, Angelov L, Koyfman SA, Suh JH, Reddy CA, Djemil T, et al. Single-fraction stereotactic body radiotherapy for spinal metastases from renal cell carcinoma. J Neurosurg Spine 2012; 17: 556-64.

4. Stinauer MA, Kavanagh BD, Schefter TE, Gonzalez R, Flaig T, Lewis K, et al. Stereotactic body radiation therapy for melanoma and renal cell carcinoma: impact of single fraction equivalent dose on local control. Radiat Oncol 2011; 6: 34.

5. Emami B, Lyman J, Brown A, Coia L, Goitein M, Munzenrider JE, et al. Tolerance of normal tissue to therapeutic irradiation. Int J Radiat Oncol Biol Phys 1991; 21: 109-22.
6. Schultheiss TE. The radiation dose-response of the human spinal cord. Int $J$ Radiat Oncol Biol Phys 2008; 71: 1455-9.

7. Ryu S, Jin JY, Jin R, Rock J, Ajlouni M, Movsas B, et al. Partial volume tolerance of the spinal cord and complications of single-dose radiosurgery. Cancer 2007; 109: 628-36.

8. Sahgal A, Ma L, Gibbs I, Gerszten PC, Ryu S, Soltys S, et al. Spinal cord tolerance for stereotactic body radiotherapy. Int J Radiat Oncol Biol Phys 2010; 77: 548-53.

9. Fowler JF. The linear-quadratic formula and progress in fractionated radiotherapy. Br J Radiol 1989; 62: 679-94.

10. Chang EL, Shiu AS, Mendel E, Mathews LA, Mahajan A, Allen PK, et al. Phase $\mathrm{I} / \mathrm{II}$ study of stereotactic body radiotherapy for spinal metastasis and its pattern of failure. J Neurosurg Spine 2007; 7: 151-60.

11. Sahgal A, Ames C, Chou D, Ma L, Huang K, Xu W, et al. Stereotactic body radiotherapy is effective salvage therapy for patients with prior radiation of spinal metastases. Int J Radiat Oncol Biol Phys 2009; 74: 723-31.

12. Ahmed KA, Stauder MC, Miller RC, Bauer HJ, Rose PS, Olivier KR, et al. Stereotactic body radiation therapy in spinal metastases. Int J Radiat Oncol Biol Phys 2012; 82: e803-9.

13. Nelson JW, Yoo DS, Sampson JH, Isaacs RE, Larrier NA, Marks LB, et al. Stereotactic body radiotherapy for lesions of the spine and paraspinal regions. Int J Radiat Oncol Biol Phys 2009; 73: 1369-75.

14. Iwata H, Matsufuji N, Toshito T, Akagi T, Otsuka S, Shibamoto Y. Compatibility of the repairable-conditionally repairable, multi-target and linear-quadratic models in converting hypofractionated radiation doses to single doses. $J$ Radiat Res 2013; 54: 367-73.

15. Park C, Papiez L, Zhang S, Story M, Timmerman RD. Universal survival curve and single fraction equivalent dose: useful tools in understanding potency of ablative radiotherapy. Int J Radiat Oncol Biol Phys 2008; 70: 847-52.

16. Kirkpatrick JP, Brenner DJ, Orton CG. Point/Counterpoint. The linearquadratic model is inappropriate to model high dose per fraction effects in radiosurgery. Med Phys 2009; 36: 3381-4.

17. Song CW, Kim MS, Cho LC, Dusenbery K, Sperduto PW. Radiobiological basis of SBRT and SRS. Int J Clin Oncol 2014; 19: 570-8.

18. Song CW, Park I, Cho LC, Yuan J, Dusenbery KE, Griffin RJ, et al. Is indirect cell death involved in response of tumors to stereotactic radiosurgery and stereotactic body radiation therapy? Int J Radiat Oncol Biol Phys 2014; 89: 924-5.

19. Joiner MC, Bentzen SM. Fractionation: the linear-quadratic approach. In: Joiner M, van der Kogel A, editors. Basic Clinical Radiobiology. London: Hodder Education; 2009. p. 102-19.

20. Bilsky MH, Laufer I, Fourney DR, Groff M, Schmidt MH, Varga PP, et al. Reliability analysis of the epidural spinal cord compression scale. $J$ Neurosurg Spine 2010; 13: 324-8.

21. Ryu S, Pugh SL, Gerszten PC, Yin FF, Timmerman RD, Hitchcock YJ, et al. RTOG 0631 phase II/III study of image-guided stereotactic radiosurgery for localized (1-3) spine metastases: phase II results. Int J Radiat Oncol Biol Phys 2011; 81: S131-2.

22. Fowler JF. Alpha, beta, and surviving fraction. Int J Radiat Oncol Biol Phys 1992; 24: 188-9.

23. Fowler JF. Modelling altered fractionation schedules. BJR Supp/ 1992; 24: 187-92.

24. Bijl HP, van Luijk P, Coppes RP, Schippers JM, Konings AW, van der Kogel AJ. Unexpected changes of rat cervical spinal cord tolerance caused by inhomogeneous dose distributions. Int J Radiat Oncol Biol Phys 2003; 57: 274-81.

25. Bijl HP, van Luijk P, Coppes RP, Schippers JM, Konings AW, van Der Kogel AJ. Regional differences in radiosensitivity across the rat cervical spinal cord. Int J Radiat Oncol Biol Phys 2005; 61: 543-51.

26. Medin PM, Foster RD, van der Kogel AJ, Sayre JW, McBride WH, Solberg TD. Spinal cord tolerance to single-fraction partial-volume irradiation: a swine model. Int J Radiat Oncol Biol Phys 2011; 79: 226-32.

27. Brenner DJ. The linear-quadratic model is an appropriate methodology for determining isoeffective doses at large doses per fraction. Semin Radiat Oncol 2008; 18: 234-9.

28. Sahgal A, Weinberg V, Ma L, Chang E, Chao S, Muacevic A, et al. Probabilities of radiation myelopathy specific to stereotactic body radiation therapy to guide safe practice. Int J Radiat Oncol Biol Phys 2013; 85: 341-7.

29. Gerszten PC, Burton SA, Ozhasoglu C, Vogel WJ, Welch WC, Baar J, et al. Stereotactic radiosurgery for spinal metastases from renal cell carcinoma. $J$ Neurosurg Spine 2005; 3: 288-95. 